\title{
Synchronous and asynchronous remote exercise may improve motor and non-motor symptoms in people with Parkinson's disease during the COVID-19 pandemic
}

\author{
FELIPE B. SANTINELLI | LUCAS SIMIELI' | ELISA DE C. COSTA ${ }^{1}$ | LETICIA N. MARTELI',2 | CHIEN H. FEN ${ }^{3,4}$ | ERICA \\ TARDELLI ${ }^{3}$ | ERIKA OKAMOTO³ ${ }^{3}$ KATIA TANAKA ${ }^{3}$ | FABIO A. BARBIERI ${ }^{1}$
}

1 São Paulo State University (UNESP), School of Sciences, Graduate Program in Movement Sciences, Department of Physical Education, Human Movement Research Laboratory (MOVI-LAB), Bauru, SP, Brazil.

2 São Paulo State University (UNESP), School of Architecture, Arts and Communication (FAAC), Postgraduate Program in Design, Ergonomic and Interfaces Laboratory (LEI), Bauru, SP, Brazil. University of Lisbon (ULisboa), School of Architecture (FA), Doctoral Program in Design, Research Centre for Architecture, Urbanism and Design (CIAUD), Lisbon, Portugal.

${ }^{3}$ Associação Brasil Parkinson (ABP), São Paulo, SP, Brazil.

${ }^{4}$ Hospital das Clínicas, Faculdade de Medicina da Universidade de São Paulo (USP), São Paulo, SP, Brazil.

Correspondence to: Fabio Augusto Barbieri. Av. Eng. Luiz Edmundo Carrijo Coube, 14-01, Vargem Limpa. Bauru, SP CEP 17033-360. Phone + 55 14 3103-9612 email: fabio.barbieri@unesp.br

https://doi.org/10.20338/bjmb.v15i1.236

\section{HIGHLIGHTS}

- Perform synchronous exercise class resulted in higher amount of physical activity.

- Synchronous exercise mitigate the anxiety symptom.

- Asynchronous exercise presented lower depression symptom than no-exercise group.

- People with PD should be encouraged to perform synchronous exercise during pandemic.

\begin{tabular}{|c|c|}
\hline \\
\hline $\begin{array}{ll}\text { ABBREVIATIONS } \\
\text { ABP } & \text { Associacão Brasileira de }\end{array}$ & $\begin{array}{l}\text { Associação Brasileira de } \\
\text { Parkinson }\end{array}$ \\
\hline FOG & Freezing of gait \\
\hline HAD & Hospital Anxiety and Depression \\
\hline IPAQ & $\begin{array}{l}\text { International Physical Activity } \\
\text { Questionnaire }\end{array}$ \\
\hline MET & Metabolic Equivalent of Task \\
\hline MSQ & Mini Sleep Questionnaire \\
\hline PD & Parkinson's Disease \\
\hline DQ-8 & Parkinson's Disease \\
\hline & Questionnaire-8 \\
\hline Q-39 & Parkinson's Disease \\
\hline & Questionnaire-39 \\
\hline SARS-Cov-2 & $\begin{array}{l}2 \text { Severe acute respiratory } \\
\text { syndrome }\end{array}$ \\
\hline
\end{tabular}

PUBLICATION DATA

Received 04012021

Accepted 27022021

Published 01032021
BACKGROUND: Stay active is a good strategy to mitigate the negative effects of confinement in people with Parkinson's Disease (PD). Synchronous (full-time class interaction) and asynchronous (without the live presence of the healthcare professional) exercises are two strategies to avoid the worsening of PD.

AIM: To investigate the effect of the synchronous and asynchronous exercises on motor and non-motor symptoms, physical activity level, anxiety, depression, sleep quality, and quality of life in people with PD during the pandemic lockdown.

METHOD: Fifty-eight people with PD responded to an online survey and were divided into synchronous $(n=24)$, asynchronous $(n=19)$, and no-exercise $(n=15)$ groups. The participants responded to questions regarding motor and non-motor symptoms, besides the questionnaire of quality of life, physical activity, anxiety and depression, and sleep quality.

RESULTS: Synchronous group presents higher amounts of physical activity than the asynchronous and noexercise groups. Also, the synchronous group presented lower anxiety symptoms, while the asynchronous group presented lower depression symptoms compared with the no-exercise group. Worse motor symptoms were presented by the three groups.

CONCLUSION: Stay active during the pandemic lockdown, is beneficial to reduce anxiety and depression symptoms in people with PD. This population should be encouraged to perform synchronous classes to perform greater amounts of physical activity, which in the long-term could produce greater benefits.

KEYWORDS: Motor control | Parkinson's disease | COVID-19 | Exercise | Depression | Motor symptoms

\section{INTRODUCTION}

The severe acute respiratory syndrome - SARS-Cov-2 (COVID-19), which caused a worldwide pandemic, altered the habits of people. Millions of people were infected with COVID-19, resulting in millions of deaths. Specifically in Brazil, more than 13 million were infected and more than 330 thousand died (data from April 05, 
https://www.worldometers.info/coronavirus/country/brazil/). To control the rate of transmission and avoid healthy system breakdown, local governments, including the Brazilian government, declared full/partial lockdowns or restricted people circulation, allowing only essential activities. These restrictions increase the time spending at home working, watching TV, or only sitting, and one consequence was the reduction of the amount of exercise.1,2 This reduction of time expending doing exercise results in an increase in mortality during pandemic lockdown. ${ }^{3}$ Thereby, an exercise routine may be a good strategy to prevent neurological ${ }^{2}$ and cardiovascular ${ }^{4}$ disease progression and should be encouraged during home confinement.

Regular practices of physical activity delay Parkinson's disease (PD) progression. ${ }^{5}$ It is well-established that higher levels of physical activity improve PD-related symptoms. ${ }^{6}$ On the other hand, lower level of physical activity increases motor (e.g., rigidity, tremor, akinesia, bradykinesia, unbalance, freezing of gait) and non-motor (e.g., depression, anxiety, sleep disturbance, fatigue) symptoms. ${ }^{7}$ Thus, keeping physically active can be a good strategy to avoid the negative effects of routine changes due to the COVID-19 pandemic in people with PD. Social distancing and home confinement hindered access to healthcare professionals, requiring alternative strategies such as home-based remotely exercise. A bunch of groups have promoted live (synchronous class) or recorded exercise (asynchronous class) classes during pandemic lockdown to promote exercise among the population, including people with PD. The synchronous class has full-time interaction between the healthcare professional and the individual, with controlled intensity prescribed and with real-time feedback and patient's movement correction.8,9 Although the asynchronous class allows the participant to perform the activity at any time of the day, it is limited or not present any kind of interaction with the class professor during the performance of class. ${ }^{9,10}$ This limited interaction with the patients could influence in some of training parameters such as the effort of the session. For instance, in longitudinal terms asynchronous exercise present lower training load values than synchronous exercise, ${ }^{11}$ which could affect some workload outcomes-dependents.

Considering that i) pandemic COVID-19 increased the time at home without exercise, ii) the lack of exercise worse motor and non-motor symptoms in people with PD, and iii) remotely strategies are the unique safe exercise to avoid COVID-19 infection, we investigated as synchronous and asynchronous remotely exercise during pandemic COVID-19 affected the motor and non-motor symptoms, physical activity level, anxiety, depression, sleep quality, and quality of life during home confinement in people with PD in the first three months of COVID-19 pandemic (March to June of 2020). We expected that both groups that performing remotely exercise present lower motor and non-motor symptoms than the no-exercise group. Besides, the group performing synchronous exercise would present lower manifestations of motor and non-motor symptoms and a higher amount of physical activity performed than the asynchronous exercise group. For the last, we expected that higher levels of physical activity, independent of the group, will be related with lower PD-related symptoms.

\section{METHODS}

\section{Participants}

Individuals with PD were invited by social media $\left(\right.$ Facebook $^{\circledR}$, Instagram $\left.{ }^{\circledR}\right)$, e-mail, 
and WhatsApp ${ }^{\circledR}$ to fill an electronic survey (Google ${ }^{\circledR}$ Forms). One hundred seven people with PD responded to this survey. The following inclusion criteria were applied: (i) diagnosis of PD confirmed by contact with the patient (e.g., social media) or the institution that they are involved in (e.g., ATIVA PARKINSON, Associação Brasileira de Parkinson $A B P)$; (ii) over 35 years old. The exclusion criteria were inadequate questionnaire answers (e.g., no answered questions) and not answer the type of exercise was performed (synchronous exercise, asynchronous exercise, or no exercise). The research was approved by University's local Ethical Committee and all individuals consented to participate in the study (\#32134620.0.0000.5398).

After the inclusion and exclusion criteria check, 58 people with PD were included in the study. The individuals were distributed into three groups according to exercise type: i) synchronous exercise group: individuals with PD who performed a live exercise for at least for two months; ii) asynchronous exercise group: individuals with PD that was performing a recorded exercise for at least two months; iii) no-exercise group: individuals with PD that no performed exercise in the two last months.

\section{Study protocol}

This is an exploratory and descriptive study. An electronic survey produced in Google ${ }^{\circledR}$ forms was filled by the participants of the study during June of 2020 . The survey has six sections about 1) general information: demographic (e.g., height, weight, and severity of the PD) and general COVID-19 information (e.g. perception, diagnostic of COVID-19 and time in lockdown- only allowed essential services); 2) impact on motor and non-motor symptoms: questions about the effects of home confinement on PD motor (motor worse, presence of tremor, freezing of gait, unbalance, bradykinesia, and experience with falls) and non-motor (loneliness, forgetfulness, fatigue and if sleep quality have been changed) symptoms; 3) quality of life: Parkinson's Disease Questionnaire-8 (PDQ-8);12 4) level of physical activity: a short version of International Physical Activity Questionnaire (IPAQ- short version); ${ }^{13} 5$ ) anxiety and depression: Hospital Anxiety and Depression (HAD);14,15 6) quality of sleep: Mini Sleep Questionnaire (MSQ). ${ }^{16}$ The participants answered the questionary from June $15^{\text {th }}$ to June $30^{\text {th }}$ of 2020 .

\section{Quality of life, level of physical activity, anxiety, depression, and quality of sleep assessments}

Several questionnaires were used to the quality of life, level of physical activity, anxiety, depression, and quality of sleep evaluation. Firstly, the PDQ-8, a shortened version of the Parkinson's Disease Questionnaire-39 (PDQ-39), a scale that assesses the quality of life of these individuals, was answered by the participants. The total score ranges from 0 to 100 , where $0=$ no problem and $100=$ maximum problem level. ${ }^{12}$ Secondly, the amount of physical activity performed during the lockdown was obtained through the IPAQ, ${ }^{13}$ which is reported here as Metabolic Equivalent of Task (MET). Thirdly, to evaluate the anxiety and depression aspects the HAD questionnaire was applied, being a score between 8 and 10 interpreted as possible cases of anxiety and depression and a score equal or greater than 11, indicates the likely presence of anxiety and depression, more significantly. ${ }^{14,15}$ Fourthly, and finally, the sleep quality was measure with the MSQ. The total score is divided into levels of difficulty sleeping being: good sleep quality (10 to 24 
points); mild difficulty (25 to 27 points); moderate difficulty (28 to 30 points); severe difficulty sleeping (31 points or more)..$^{16-18}$

\section{Data and statistical analysis}

The demographic characteristics, and self-reported motor and non-motor symptoms were reported in Table 1, 2, and 3, respectively. The demographic characteristics were expressed in the average of the absolute values, while self-reported motor and non-motor symptoms were presented as a percentage. The level of physical activity (IPAQ), quality of life (PDQ-8), quality of sleep (MSQ), anxiety, and depression (HAD) were compared among groups using univariate analysis (synchronous exercise vs asynchronous exercise vs no-exercise). Tukey post hoc, with significant levels adjusted, was used when ANOVA showed a significant effect. We also performed a correlation analysis, through Spearman rank correlation, between the IPAQ with the PDQ-8, MSQ, HAD-anxiety, and HAD-depression. Correlation coefficients of $0.1,0.3$, and 0.5 were interpreted as weak, moderate, and strong, respectively. ${ }^{19}$ All analyses were conducted using SPSS software version 26 (IBM Corporation, Armory, NY) and significance was set at $p<0.05$.

\section{RESULTS}

\section{Demographic}

Table 1 presents the demographic data. Only one subject of the non-exercise group maybe was positive for COVID-19. The majority of the participants from both groups maintained the habitual PD medicine, and the asynchronous group was more time in lockdown (more than 7 weeks- 94.7\%) than the synchronous group (79.2\%), and nonexercise group (80\%). The majority of the participants from the three groups live with someone (more than 80\%).

\section{Motor symptoms}

The answers for motor symptoms are presented in Table 2. The majority of the participants observed worsening in motor symptoms during the lockdown period (asynchronous: $63 \%$, synchronous: $79 \%$, and no-exercise: $73 \%$ - total: $72.4 \%$ ). Noexercise group presented tremor symptoms and imbalance (46.7\% in both symptoms) more often than both asynchronous $(26.4 \%$ and $36.8 \%$, respectively) and synchronous (37.5\% and $29.2 \%$, respectively) groups, but they had less often (13.3\%) freezing of gait compared to synchronous (33.3\%) and asynchronous (26.3\%) groups. Finally, falls were experienced by the three groups, with a higher percentage in the asynchronous (47.4\%) group compared to both no-exercise $(33.3 \%)$ and synchronous $(29.2 \%)$ groups.

\section{Non-motor symptoms}

Table 3 presents the answers for non-motor symptoms. The synchronous group feels lesser isolated (50\%) compared to asynchronous (26.3\%) and no-exercise $(33.3 \%)$ groups. Forgetfulness was perceived worse for the asynchronous (31.6\%) and synchronous $(37.5 \%)$ than the no-exercise (20\%) group. Individuals in the no-exercise group (46.7\%) reported lower sleep quality than asynchronous (36.8\%) and synchronous (25\%) groups. 
Table 1 - Anthropometric characteristics, disease record education level and aspects related to the COVID-19 pandemic lockdowns such as time in isolation and seriousness of the pandemic.

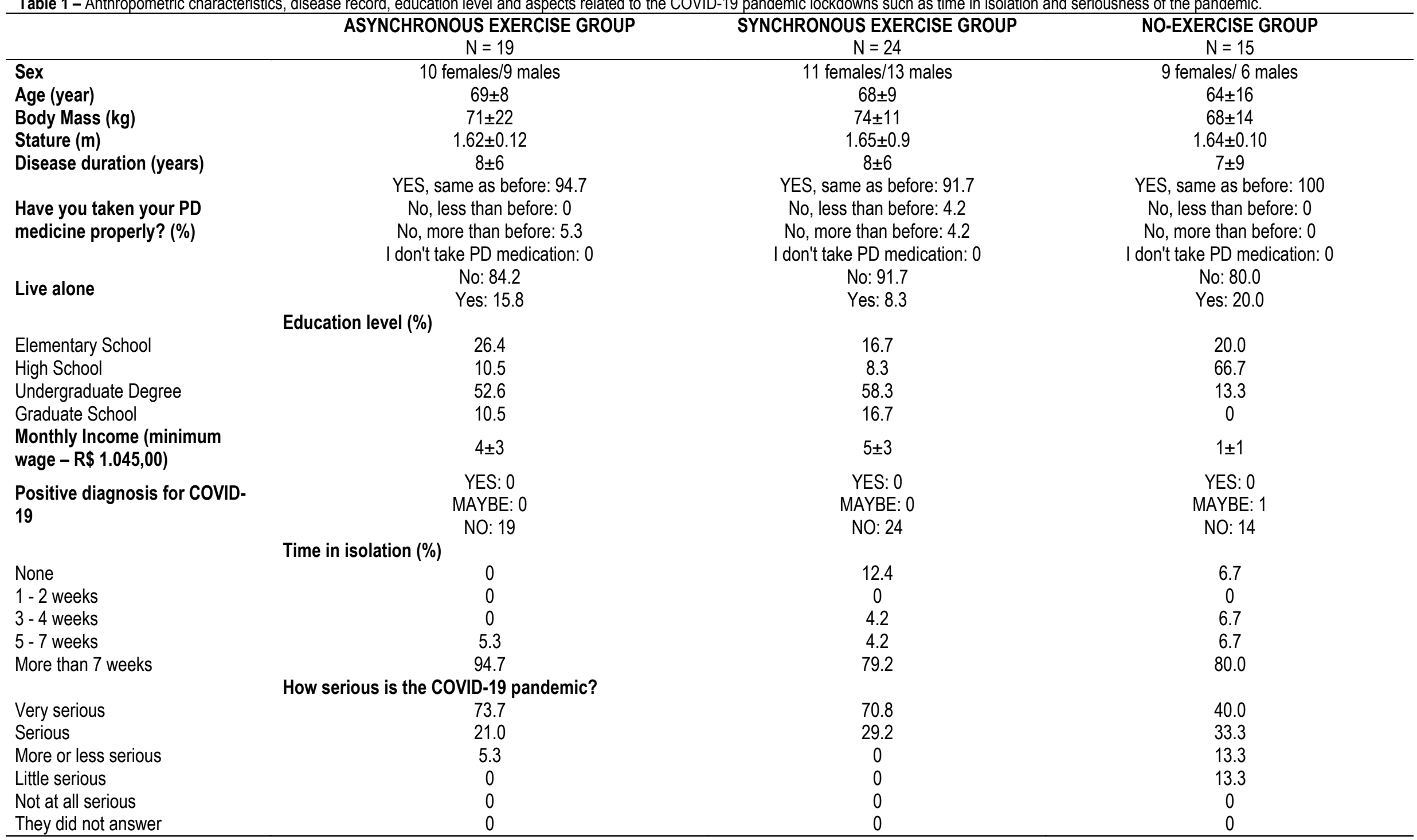


Table 2 - Self-reported motor symptoms during the quarantine for the asynchronous, synchronous, and no-exercise groups.

\section{ASYNCHRONOUS EXERCISE}

Did you notice any motor
worsening? (\%)
Did you experience tremor at
rest? (\%)

\section{You presented freezing of gait?}

(\%)

\section{You presented imbalance? (\%)}

Were your movements slower?

Did you have falls? (\%)

YES: 63.0
NO: 37.0
NO: 36.8

YES, more often: 26.4

YES, with the same frequency: 36.8

YES, less frequently: 0

They did not answer: 0

NO: 57.9

YES, more often: 26.3

YES, with the same frequency: 10.5

YES, less frequently: 5.3

They did not answer: 0

$$
\text { NO: } 31.6
$$

YES, more often: 36.8

YES, with the same frequency: 31.6

YES, less frequently: 0

They did not answer: 0

YES: 63.2
NO: 36.8

YES: 47.4

NO: 52.6

\section{SYNCHRONOUS}

NO-EXERCISE

YES, more often: 37.5

YES, with the same frequency: 16.7

YES, less frequently: 0

They did not answer: 0

NO: 41.7

YES, more often: 33.3

YES, with the same frequency: 12.5

YES, less frequently: 4.2

They did not answer: 8.3

$$
\text { NO: } 37.5
$$

YES, more often: 29.2

YES, with the same frequency: 33.3

YES, less frequently: 0

They did not answer: 0

YES: 79.0
NO: 21.0
NO: 45.8

YES: 73.0
NO: 27.0
NO: 6.7

Yes, more often: 46.7

Yes, with the same frequency: 46.7

Yes, less frequently: 0

They did not answer: 0

NO: 66.7

YES, more often: 13.3

YES, with the same frequency: 20.0

YES, less frequently: 0

They did not answer: 0

NO: 33.3

YES, more often: 46.7

YES, with the same frequency: 20.0

YES, less frequently: 0

They did not answer: 0

YES: 60.0

NO: 40.0

YES: 58.3

NO: 41.7

YES: 29.2

NO: 70.8
YES: 33.3

NO: 66.7 
Table 3 - Self-reported non-motor symptoms during the quarantine for the asynchronous, synchronous, and no-exercise groups.

\section{ASYNCHRONOUS EXERCISE}

$$
\text { NO: } 26.3
$$

YES, more often: 54.1

Do you feel isolated (loneliness)? (\%) (\%)

Have you been tired (fatigue)? (\%)

Has your sleep quality changed? (\%)
YES, with the same frequency: 15.8

YES, less frequently: 3.8

They did not answer: 0

NO: 21.0

YES, more often: 31.6

YES, with the same frequency: 47.4

YES, less frequently: 0

They did not answer: 0

\section{NO: 36.8}

YES, more often: 31.6

YES, with the same frequency: 31.6

YES, less frequently: 0

They did not answer: 0

NO: 52.6

YES, it got worse: 36.8

YES, it improved: 10.5

They did not answer: 0

SYNCHRONOUS

\section{NO-EXERCISE}

\section{NO: 50}

YES, more often: 33.3

YES, with the same frequency: 8.3

YES, less frequently: 2.0

They did not answer: 4.2

NO: 29.2

YES, more often: 37.5

YES, with the same frequency: 29.2

YES, less frequently: 4.2

They did not answer: 0

NO: 25.0

YES, more often: 45.8

YES, with the same frequency: 25.0

YES, less frequently: 4.2

They did not answer: 0

NO: 58.3

YES, it got worse: 25.0

YES, it improved: 12.5

They did not answer: 4.2
NO: 33.3

YES, more often: 66.7

YES, with the same frequency: 0

YES, less frequently: 0

They did not answer: 0

NO: 60.0

YES, more often: 20.0

YES, with the same frequency: 20.0

YES, less frequently: 0

They did not answer: 0

NO: 40.0

YES, more often: 40.0

YES, with the same frequency: 20.0

YES, less frequently: 0

They did not answer: 0

NO: 53.3

YES, it got worse: 46.7

YES, it improved: 0

They did not answer: 0 
Physical activity, depression and anxiety level, quality of sleep, and quality of life

ANOVA indicated significant difference for level of physical activity $\left(F_{2,58}=5.73\right.$, $p<0.005)$, anxiety $\left(F_{2,58}=3.40, p<0.04\right)$ and depression $\left(F_{2,58}=3.26, p<0.04\right)$ (Figure 1). Post hoc analysis showed that the synchronous exercise group had a higher physical activity level than both asynchronous exercise $(p<0.05)$ and no-exercise $(p<0.007)$ groups. Also, the no-exercise group presented higher anxiety than the synchronous exercise group $(p<0.04)$ and higher depression levels compared with the asynchronous exercise group $(p<0.05)$.
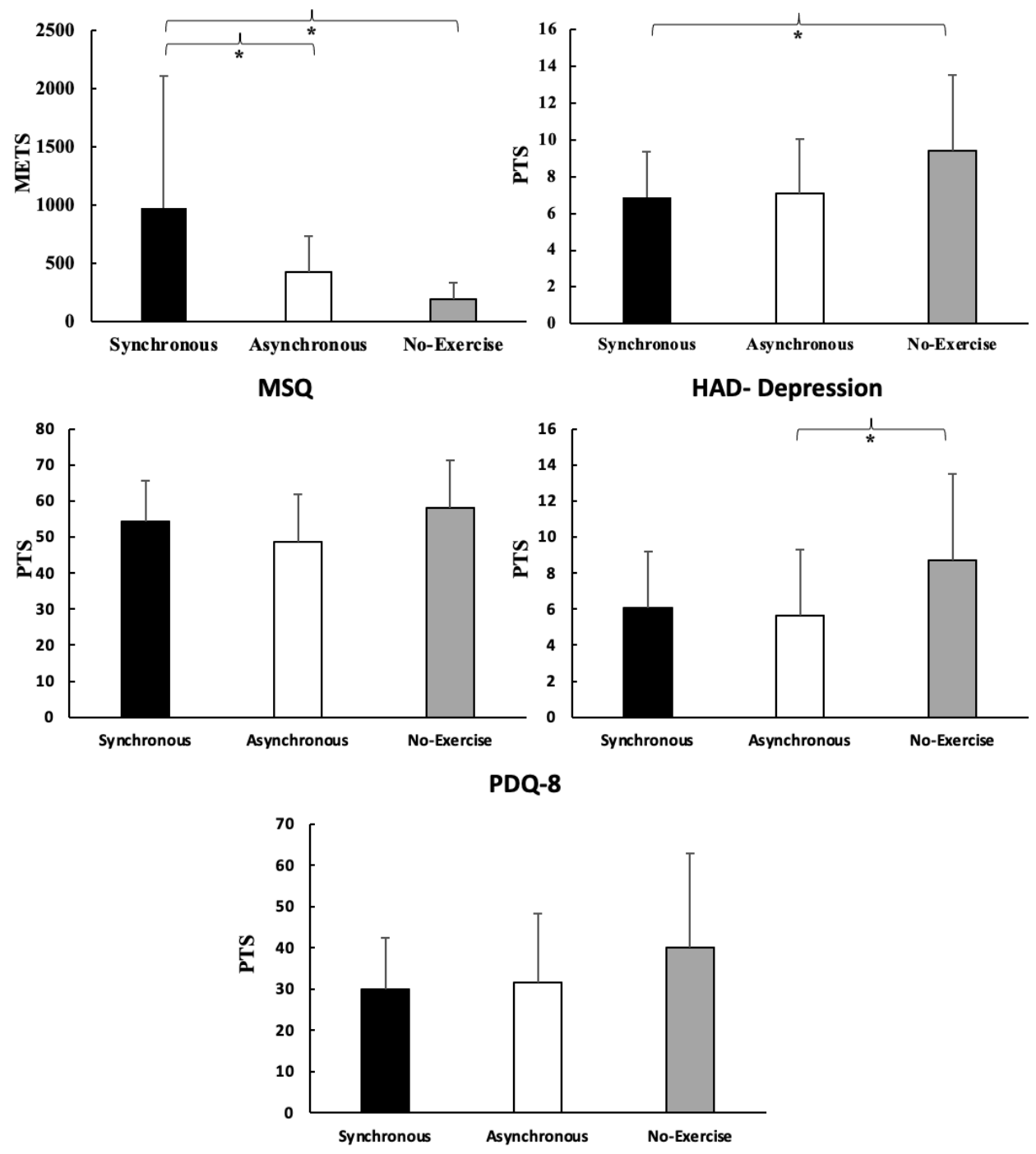

Figure 1. Means and standard deviation of level of physical activity (IPAQ), anxiety (HAD), sleep quality (MSQ), depression (HAD), and quality of life (PDQ-8). * Significant differences between groups.

\section{Correlation's analysis}

Higher levels of physical activity, obtained by the IPAQ, was significant correlated with lower depression (moderate relationship) and with anxiety (weak relationship) 
symptoms considering the three groups. No significant correlations were observed between the IPAQ and sleep quality (MSQ) and quality of life (PDQ-8).

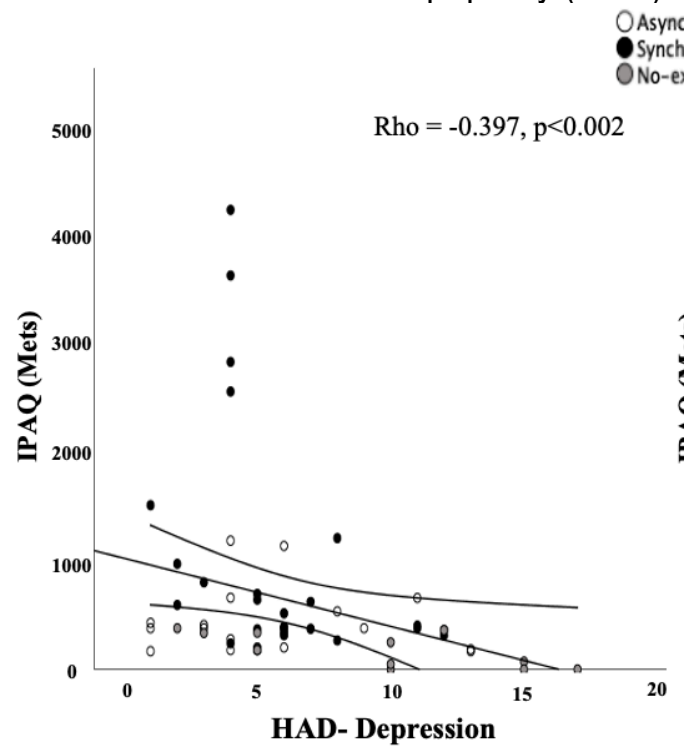

Asynchronous

Synchronous
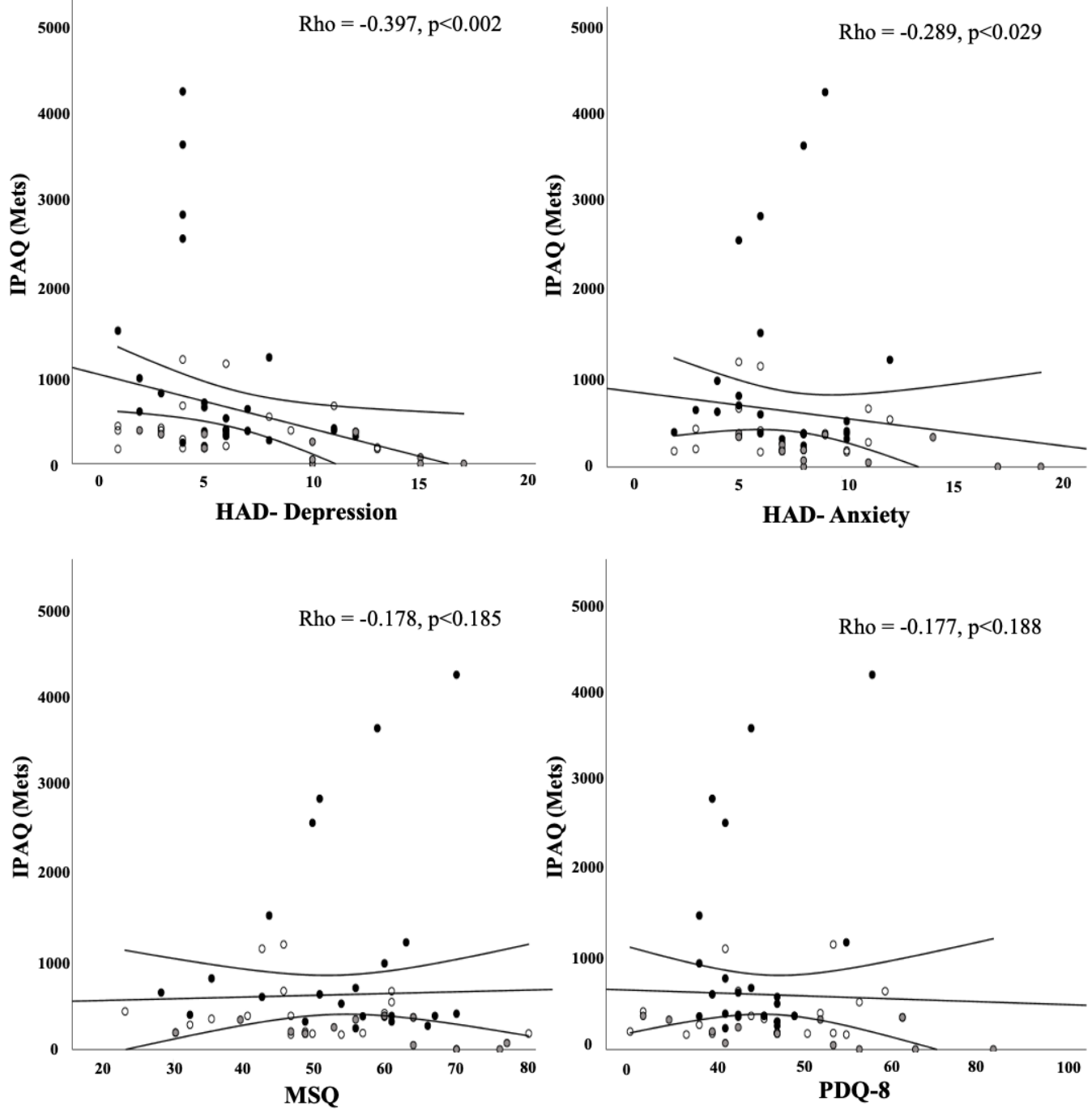

Figure 2. Correlations between the physical activity questionnaire (IPAQ), anxiety (HAD-anxiety), sleep quality (MSQ), depression (HAD-depression), and quality of life (PDQ-8). The curved line represents the $95 \%$ confidence intervals.

\section{DISCUSSION}

In the present study, we investigated the impact of synchronous exercise, asynchronous exercise, and no performance of exercise on the motor and non-motor symptoms, physical activity level, anxiety, depression, sleep quality, and quality of life in people with PD during the pandemic lockdown. The main findings of the study were: i) the three groups presented worsens in symptoms during quarantine of COVID-19 pandemic lockdown, with the no-exercise group presenting more often worsens in tremor, imbalance and sleep symptoms, ii) synchronous exercise presented higher amount of physical activity compared to asynchronous and no-exercise groups, iii) synchronous exercise reduced 
levels of anxiety and asynchronous exercise reduced levels of depression compared to the no-exercise group, iv) Higher levels of physical activity were associated with lower anxiety and depression symptoms in people with PD. The results confirmed in part our hypothesis. Our results suggest that that performing exercise remotely, under or not supervision, is beneficial for motor and non-motor symptoms in people with PD during pandemic COVID19 lockdown, improving the level of physical activity.

Sustain higher amounts of physical activity, mainly during pandemic COVID-19 lockdown, is essential for the maintenance of health in people with PD. In an Italian survey during the pandemic lockdown, $62.3 \%$ of the people with PD reported that they did not perform any kind $(42.3 \%)$ or poor $(20 \%)$ physical activity, while only $37.6 \%$ responded that only home-based physical activity was performed. ${ }^{1}$ In another Italian survey, Schirinzi et al. ${ }^{2}$ observed that the patients which perceived worsening during pandemic lockdown ( $60 \%)$, performed a lower amount of physical activity (1714 \pm 1570 METS) vs those who not reported worsening of motor symptoms $(2399 \pm 2412$ METS), indicating that the amount of physical activity performed is the main risk factor associated with the perception of worsening of motor symptoms. In our study, the amount of physical activity observed was lower compared with the Schirinzi et al. ${ }^{2}$ study, even in the synchronous group (1057 \pm 1142 METS). This explains why most of our cohort reported, in general, worsening of motor and non-motor symptoms. However, the no-exercise group showed worsen balance and tremor compared to synchronous and asynchronous exercise groups during the pandemic lockdown, which could indicate some exercise benefits on those aspect. Nevertheless, both exercise groups presented more recurrence of freezing of gait (FOG) symptom than no-exercise group, but maybe for the fact that the exercise groups spending more time (i.e., greater amount of physical activity) in situations that could produce FOG (e.g., pass through a door).

The synchronous exercise group presented a significantly lower anxiety score while the asynchronous exercise group presented a lower depression score than the noexercise group. Also, although non-significant, the synchronous group presented a lower depression score and the asynchronous group presented lower anxiety compared with those who not performed exercise. It is common for people with PD to report higher levels of anxiety and depression, ${ }^{20}$ which could be exacerbated during pandemic restrictions. ${ }^{21}$ Pandemic lockdown could cause stress, anxiety, social isolation, and psychological distress ${ }^{22}$ and be associated with unpleasant emotions, sadness, anger, and frustrations. ${ }^{4}$ This worsening in mental health aspects could be related to a reduced amount of physical activity. Anyan et al. ${ }^{23}$ observed that reduced physical activity was associated with a higher risk to develop anxiety and depressive symptoms during the pandemic. It is wellestablished that physical activity is essential to decrease anxiety and depression symptoms in a range of populations, including PD. ${ }^{24}$ Confirming these previous studies, the correlations of our study showed that maintain higher levels of physical activity during confinement could ease the anxiety and depression symptoms in people with PD. Thereby, it seems that perform any kind of exercise class, synchronous or not, seems to be beneficial to improve mental health during the pandemic lockdown. However, the amount of physical activity seems to change between the two types of exercise class and with those who have not participated in either of both classes.

Performing a synchronous or asynchronous class exercise showed similar results for people with PD for motor and non-motor symptoms, but a greater amount of physical 
activity for the synchronous group was observed compared with the other two groups. During the pandemic lockdown, Gomes Costa et al. ${ }^{11}$ investigated the difference between synchronous and asynchronous classes in spinal cord injuries patients. Greater benefits for synchronous classes were observed related to total and weekly average workload and program adherence over the asynchronous classes. This corroborates with the literature. Perform exercise under professional supervision provides greater results (e.g., higher motivation, specific orientation, correction of movement, optimal frequency, intensity, and volume) when compared with exercise without supervision. ${ }^{25}$. The fact that the synchronous group in our study presented higher amounts of physical activity may suggest some of these benefits performing exercise under supervision (e.g., greater adherence and higher workload). In addition, we suggest that perform synchronous exercise reduced the feeling of isolation, which indicates that synchronous activities may reduce loneliness during the lockdown. This may be an important aspect considering the high number of psychological and cognitive problems reported during pandemic. ${ }^{4,22}$ Thus, given that no social interaction could increase anxiety ${ }^{26}$ and depression ${ }^{27}$ symptoms the synchronous exercise, which provide social interaction, seems to be the most recommended to mitigate these non-motor symptoms. In addition, once the synchronous exercise provides a greater amount of physical activity than asynchronous exercise and no-exercise, which may reflect a decrease in motor and non-motor symptoms in a long-term investigation.

Although some important findings were obtained in the present study, some precautions should be considered to interpret the results. First, we were not able to evaluate the participants before the pandemic lockdown, which limits our results based only on an observation. Besides, we can not to match the groups in accord with the sex which could certainly influence motor and non-motor symptoms in people with PD. 28 We propose for future studies to address these issues comparing pre and post synchronous/asynchronous class exercise exposure to confirm, or not, our findings. Thereby, it will be possible to confirm if the differences observed were indeed due to the performance of each exercise modality or due the baseline differences among groups. Second, it is not possible to confirm if the groups were matched for the PD disability level, influencing the perception of the participants related to motor and non-motor symptoms, explaining the lack of difference for some parameters among the groups. ${ }^{29}$ Third, a longitudinal study could strength our findings, confirming (or not) the benefits of synchronous or asynchronous exercise during pandemic COVID-19 lockdown. For the last, as the present study it is an observational study, we were not able to control the parameters of the exercise groups such as the type of exercise, intensity, volume, frequency or duration of each session which could influence the outcomes evaluated.

\section{CONCLUSION}

This study demonstrated that stay active, through synchronous or asynchronous exercises, during COVID-19 pandemic lockdown is beneficial for motor and non-motor symptoms, especially for anxiety and depression, in people with PD. In addition, synchronous exercise increased the level of physical activity compared to asynchronous exercises and no-exercise. Based on our survey, people with PD and their caregivers should plan for access to a remote exercise class (preference for synchronous class) to preserve or improve motor and non-motor symptoms. In the absence of the possibility to 
achieve synchronous class, these populations should be encouraged to perform any kind of physical activity to preserve health and to prevent the worse of PD.

\section{REFERENCES}

1. Cavallieri F, Sireci F, Fioravanti V, et al. Parkinson Patients' needs during COVID-19 pandemic in a red zone: a Framework Analysis of Open-Ended Survey Questions. Eur J Neurol. 2021; 1-9.

2. Schirinzi T, Di Lazzaro G, Salimei C, et al. Physical Activity Changes and Correlate Effects in Patients with Parkinson's Disease during COVID-19 Lockdown. Mov Disord Clin Pract. 2020; 7: 797-802.

3. Marçal IR, Fernandes B, Viana AA, et al. The Urgent Need for Recommending Physical Activity for the Management of Diabetes During and Beyond COVID-19 Outbreak. Front Endocrinol; 11. Epub ahead of print. 2020. 10.3389/fendo.2020.584642.

4. Lippi G, Henry BM, Sanchis-Gomar F. Physical inactivity and cardiovascular disease at the time of coronavirus disease 2019 (COVID-19). Eur. J. Prev. Cardiol. 2020; 27: 906-908.

5. Fayyaz M, Jaffery SS, Anwar F, et al. The Effect of Physical Activity in Parkinson's Disease: A Mini-Review. Cureus; 10. Epub ahead of print. 2018. 10.7759/cureus.2995.

6. Li Y, Song H, Shen L, et al. The efficacy and safety of moderate aerobic exercise for patients with Parkinson's disease: a systematic review and meta-analysis of randomized controlled trials. Ann Palliat Med; Epub ahead of print. 2021. 10.21037/apm-20-1661.

7. Nimwegen M Van, Speelman AD, Hofman-Van Rossum EJM, et al. Physical inactivity in Parkinson's disease. J Neurol. 2011; 258: 2214-2221.

8. Middleton A, Simpson KN, Bettger JP, et al. COVID-19 Pandemic and Beyond: Considerations and Costs of Telehealth Exercise Programs for Older Adults With Functional Impairments Living at Home-Lessons Learned From a Pilot Case Study. Phys Ther. 2020; 100: 1278-1288.

9. Chan S, Li L, Torous J, et al. Review of Use of Asynchronous Technologies Incorporated in Mental Health Care. Curr Psychiatry Rep; 20. Epub ahead of print. 2018. 10.1007/s11920-018-0954-3.

10. Gupta MM, Jankie S, Pancholi SS, et al. Asynchronous Environment Assessment: A Pertinent Option for Medical and Allied Health Profession Education During the COVID-19 Pandemic. Educ Sci. 2020; 10: 352.

11. Costa RRG, Dorneles JR, Veloso JHCL, et al. Synchronous and asynchronous teleexercise during the coronavirus disease. 2019 pandemic: Comparisons of implementation and training load in individuals with spinal cord injury. J Telemed Telecare. Epub ahead of print. 2021. 10.1177/1357633X20982732.

12. Jenkinson C, Fitzpatrick R, Peto V, et al. The PDQ-8: Development and validation of a short-form Parkinson's disease questionnaire. Psychol Health. 1997; 12: 805-814. 
13. Matsudo S, Araújo T, Matsudo V, et al. Questionário Internacional De Atividade Física (Ipaq): Estupo De Validade E Reprodutibilidade No Brasil. Rev Bras Ativ Fis Saúde. 2012; 6: $5-18$.

14. Andrews B, Hejdenberg J, Wilding J. Student anxiety and depression: Comparison of questionnaire and interview assessments. J Affect Disord. 2006; 95: 29-34.

15. Leentjens AFG, Verhey FRJ, Lousberg $R$, et al. The validity of the Hamilton and Montgomery-Asberg Depression Rating Scales as screening and diagnostic tools for depression in Parkinson's disease. Int J Geriatr Psychiatry. 2000; 15: 644-649.

16. Falavigna A, De Souza Bezerra ML, Teles AR, et al. Consistency and reliability of the Brazilian Portuguese version of the Mini-Sleep Questionnaire in undergraduate students. Sleep and Breath. 2011; 15: 351-355.

17. Natale V, Fabbri M, Tonetti L, et al. Psychometric goodness of the Mini Sleep Questionnaire. Psychiatry Clin Neurosci. 2014; 68: 568-573.

18. Falavigna A, De Souza Bezerra ML, Teles AR, et al. Sleep disorders among undergraduate students in Southern Brazil. Sleep and Breath. 2011; 15: 519-524.

19. Cohen J. Statistical power analysis for the behavioral sciences - 2nd ed. 1988.

20. Hemmerle AM, Herman JP, Seroogy KB. Stress, depression and Parkinson's disease. Exp Neurol. 2012; 233: 79-86.

21. Kitani-Morii F, Kasai T, Horiguchi G, et al. Risk factors for neuropsychiatric symptoms in patients with Parkinson's disease during COVID-19 pandemic in Japan. PLoS One. 2021; 16: 1-13.

22. Violant-Holz V, Gallego-Jiménez MG, González-González CS, et al. Psychological health and physical activity levels during the covid-19 pandemic: A systematic review. Int J Environ Res Public Health. 2020; 17: 1-19.

23. Anyan F, Hjemdal O, Ernstsen L, et al. Change in Physical Activity During the Coronavirus Disease 2019 Lockdown in Norway: The Buffering Effect of Resilience on Mental Health. Front Psychol. 2020; 11: 1-8.

24. Carek PJ, Laibstain SE, Carek SM. Exercise for the treatment of depression and anxiety. Int J Psychiatry Med. 2011; 41: 15-28.

25. Lacroix A, Hortobágyi T, Beurskens R, et al. Effects of Supervised vs. Unsupervised Training Programs on Balance and Muscle Strength in Older Adults: A Systematic Review and Meta-Analysis. Sports Med. 2017; 47: 2341-2361.

26. Santini ZI, Jose PE, York Cornwell E, et al. Social disconnectedness, perceived isolation, and symptoms of depression and anxiety among older Americans (NSHAP): a longitudinal mediation analysis. Lancet Public Health. 2020; 5: e62-e70.

27. Elmer $T$, Stadtfeld $C$. Depressive symptoms are associated with social isolation in face-toface interaction networks. Sci Rep. 2020; 10: 1-12.

28. Lubomski M, Louise Rushworth R, Lee W, et al. Sex differences in Parkinson's disease. J Clin Neurosci. 2014; 21: 1503-1506. 
29. Hitchcott PK, Fastame MC, Corona F, et al. Self-reported physical and mental health and motor functioning in elders with and without Parkinson's disease. Psychol Health Med. 2019; 24: 788-797.

\section{ACKNOWLEDGEMENTS}

The authors would like to thank all the participants to contribute with this research.

Citation: Santinelli FB, Simieli L, Costa EC, Martineli LN, Fen CH, Tardelli E, Okamoto E, Tanaka K, Barbieri FA. Synchronous and asynchronous remote exercise may improve motor and non-motor symptoms in people with Parkinson's disease during the COVID-19 pandemic. BJMB. 2021:15(1): 47-60.

Editors: Dr Fabio Augusto Barbieri - São Paulo State University (UNESP), Bauru, SP, Brazil; Dr José Angelo Barela São Paulo State University (UNESP), Rio Claro, SP, Brazil; Dr Natalia Madalena Rinaldi - Federal University of Espírito Santo (UFES), Vitória, ES, Brazil.

Copyright:@ 2021 Santinelli, Simieli, Costa, Martinelli, Fen, Tardelli, Okamoto, Tanaka and Barbieri and BJMB. This is an open-access article distributed under the terms of the Creative Commons Attribution-Non Commercial-No Derivatives 4.0 International License which permits unrestricted use, distribution, and reproduction in any medium, provided the original author and source are credited.

Funding: This research did not receive any specific grant from funding agencies in the public, commercial, or not-forprofit sectors.

Competing interests: The authors have declared that no competing interests exist.

DOI: https://doi.org/10.20338/bjmb.v15i1.236 